\title{
Value of exchangeable electrolyte measurement in the treatment of myasthenia gravis
}

\author{
MAIR CRITCHLEY, K. J. HERMAN, M. HARRISON, R. A. SHIELDS, AND \\ L. A. LIVERSEDGE
}

From the Departments of Nuclear Medicine, Medical Physics and Neurology, Manchester Royal Infirmary, Manchester

SUMMARY We have investigated electrolyte exchange in myasthenia gravis before and after corticotrophin therapy, using a four-isotope dilution technique. Clinical deterioration in patients on corticotrophin is associated with a fall of more than $20 \%$ in the intracellular potassium concentration, suggesting that all myasthenics so treated should be given potassium supplementation.

Myasthenia gravis is a disease of the neuromuscular junction characterised by excessive fatigue and weakness of voluntary muscles after repetitive activation, with recovery at rest. It has been suggested that acetylcholine activity at the myoneural junction is modified by immunological factors in which a thymic hormone may play some part.

Treatment with anticholinesterase drugs is frequently supplemented with corticotrophins which suppress the autoimmune activity of the thymus gland. The value of such corticotrophin therapy in myasthenia gravis is well documented but in the early days of treatment some patients deteriorate clinically and may require assisted ventilation and tracheostomy (Liversedge et al., 1974). Sodium intake is restricted to $20 \mathrm{mEq}$ per day. The risk of deterioration appeared to be lessened by dietary restriction of sodium and by potassium supplementation $^{1}$ but this treatment is empirical and hitherto it has not been possible to determine why some of the myasthenic patients deteriorate when treated with corticotrophins. The purpose of this communication is to investigate the electrolyte exchange before and after corticotrophin therapy by a four-isotope dilution technique and so try to correlate electrolyte changes with the clinical course.

\section{Theory of investigation}

Let $V_{H}$ be the volume of dilution of tritiated water $\mathrm{V}_{\mathrm{Br}}$ be the volume of dilution of bromide ions $\mathrm{V}_{\mathrm{K}}$ be the volume of dilution of potassium ions

${ }^{1}$ Potassium is administered in the form of Slow $\mathrm{K}$, one tablet six times daily; this amounts to a total of $48 \mathrm{mEq}$ per day.

Accepted 20 October 1976
$(\mathrm{Kp})$ be the chemical concentration of potassium in plasma and $(\mathrm{Ku})$ be the chemical concentration of potassium in urine.

Now intracellular fluid volume $=$ Total body water $\stackrel{\overrightarrow{0}}{\overrightarrow{0}}$ U extracellular fluid volume

$$
=\mathrm{V}_{\mathrm{H}}-\mathrm{V}_{\mathrm{Br}}
$$

Total extracellular potassium $=\mathrm{V}_{\mathrm{Br}} \times(\mathrm{Kp})$

Total exchangeable potassium $=\mathrm{V}_{\mathbf{K}} \times(\mathrm{Ku})$

Intracellular potassium concentration

$$
\begin{gathered}
\frac{\frac{\text { III }- \text { II }}{I}}{I} \\
\frac{V_{K} \times(K u)-V_{B r} \times(K p)}{V_{H}-V_{B r}}
\end{gathered}
$$

\section{Methods}

Eleven patients with myasthenia gravis and five selected control subjects volunteered for the test. Their renal and gastrointestinal functions were normal. We adopted the same protocol for all patients.

After a fast for four hours, a venous blood sample was analysed and corrections made for any previous isotope administration. The patients then drank a four-isotope cocktail containing $20 \mu \mathrm{Ci}{ }^{24} \mathrm{Na}, 50 \mu \mathrm{Ci}$ ${ }^{43} \mathrm{~K}, 30 \mu \mathrm{Ci}{ }^{77} \mathrm{Br}$, and $200 \mu \mathrm{Ci}{ }^{3} \mathrm{H}_{2} \mathrm{O}$, after which they resumed a normal diet. A 23 hour urine collection was made and at 24 hours venous blood and 'spot' urine samples taken. The patients fasted, from the 20th to the 24th hour.

After centrifugation of the blood samples and measurement of 'pooled' and 'spot' urine volumes, chemical concentrations of sodium and potassium were measured. 
An anion ion exchange column through which aliquots of all the samples passed, removed the bromide ions before estimation of ${ }^{24} \mathrm{Na}$ and ${ }^{43} \mathrm{~K}$ concentrations in an automatic dual channel well scintillation counter. Similarly, aliquots passing through a cation ion exchange column allowed estimation of ${ }^{77} \mathrm{Br}$ concentration in the scintillation counter.

Tritiated water samples, prepared by passing aliquots through a mixed-bed cation/anion ion exchange resin to remove all other isotopes, were counted in a liquid scintillation counter after quench correction.

Intracellular potassium concentration was then determined as in equation IV and the test repeated 14 days later, after a five day course of $1 \mathrm{mg}$ intramuscular tetracosactrin (Synacthen), a synthetic corticotrophin. On this occasion, $400 \mu \mathrm{Ci}$ of tritiated water was given.

Careful clinical assessment of the patients' muscular power and fatiguability was carried out during and after therapy and daily estimates of pulmonary vital capacity recorded using a Wright peak flow meter.

\section{Results}

Three of the eleven patients with myasthenia gravis became much worse on tetracosactrin therapy and required admission to the respiratory care unit. Their intracellular potassium concentration decreased by more than $20 \%$ from the initial pretreatment level (Table). By contrast, the intracellular potassium concentration did not fall so

Table Clinical response and percentage change in intracellular potassium concentration before and after tetracosactrin

\begin{tabular}{|c|c|c|c|}
\hline Patient & Diagnosis & $\begin{array}{l}\text { Clinical response } \\
\text { on tetracosactrin }\end{array}$ & $\begin{array}{l}\text { Percentage } \\
\text { change in } \\
\text { intracellular } \\
\text { K conc. }\end{array}$ \\
\hline 1 & Myasthenia gravis & $\begin{array}{l}\text { Poor initially } \\
\text { then v. good }\end{array}$ & -17 \\
\hline 2 & Myasthenia gravis & $\begin{array}{l}\text { Poor initially } \\
\text { then v. good }\end{array}$ & -10 \\
\hline 3 & Myasthenia gravis & Unchanged & ISQ \\
\hline 4 & Myasthenia gravis & Good & -14 \\
\hline 5 & Myasthenia gravis & Good & +13 \\
\hline 6 & Myasthenia gravis & Good & -2 \\
\hline 7 & Myasthenia gravis & Good & +29 \\
\hline 8 & Myasthenia gravis & Poor & -38 \\
\hline 9 & Myasthenia gravis & Poor & -22 \\
\hline 10 & Myasthenia gravis & Poor & -23 \\
\hline 11 & Myasthenia gravis & Good & -3 \\
\hline 12 & Multiple sclerosis & Unchanged & -2 \\
\hline 13 & Multiple sclerosis & Unchanged & $-1 \overline{3}$ \\
\hline 14 & Multiple sclerosis & Unchanged & -6 \\
\hline 15 & $\begin{array}{l}\text { Acute myeloid } \\
\text { leukaemia }\end{array}$ & Unchanged & -8 \\
\hline
\end{tabular}

precipitously in the six myasthenics who responded well. Patients 1 and 2 responded poorly initially but then improved considerably. Patients 5 and 7 were unusual. Their intracellular potassium concentration increased after therapy. Patient 5 had a crisis 10 minutes before the second set of investigations, while patient 7 was inadvertently given slow potassium supplementation during the course of her therapy with tetracosactrin.

It must be emphasised there was no correlation between clinical response and levels of serum $\mathrm{Na}, \mathrm{K}$, extracellular sodium, or the percentage of $\mathrm{Na}$ and $\mathrm{K}$ excreted.

Of the four control volunteers, three suffered from multiple sclerosis and the remaining patient had acute myeloid leukaemia. In none of these patients did the intracellular potassium concentration fall by more than $20 \%$ after therapy. Earlier estimations in our laboratory have shown that the normal intracellular potassium concentration is $128 \mathrm{mmol} / \mathrm{l}$. The estimated SD on a single determination of intracellular potassium concentration is $5.5 \%$.

\section{Discussion}

The simultaneous measurement of exchangeable sodium, potassium, extracellular fluid volume, and total body water not only gives information about body stores but also permits calculation of distribution of electrolytes and water between intra- and extracellular compartments and of intracellular concentration of sodium and potassium.

The determination of serum potassium concentration in neuromuscular disease is of little help to the clinician, for the values obtained have been almost uniformly within the normal range. Likewise, potassium balance studies, though suggesting gains or losses of potassium in the body over a period of time, give no indication of the total amount present in the body. Since $95 \%$ of the potassium in the body is intracellular, neither serum values nor balance studies will reflect intracellular derangement of potassium metabolism. Exchangeable potassium and its derived value, intracellular potassium, appear to be more sensitive indicators of redistribution of body stores.

Intracellular electrode studies of the physiological properties of the neuromuscular junction in isolated intercostal muscles of normal and of myasthenic patients have shown that the resting membrane potential of single muscle fibres is $70-90 \mathrm{mV}$ (Dählback et al., 1961). We have used data from the isotope studies to calculate change in membrane potentials, using the Nernst equation relating potassium concentration inside and outside the cell membrane. 
Membrane potential $=61 \log _{10} \frac{(\mathrm{K}+) \text { outside }}{(\mathrm{K}+) \text { inside }}$

Our values so calculated lie between 70 and $90 \mathrm{mV}$.

There was no correlation between change in the patients' condition and changes in the membrane potential after tetracosactrin therapy.

The present study, while non-invasive and atraumatic for the patient, gives a radiation dose of 240 mrads, equivalent to three chest radiographs.

Our results suggest that, because clinical deterioration in myasthenia gravis treated with corticotrophin is associated with a fall of more than $20 \%$ in the intracellular potassium concentration, all myasthenic patients so treated should be given potassium supplementation.

We wish to thank all the patients who kindly agreed to take part in this study.

\section{References}

Liversedge, L. A., Yuill, G. M., Wilkinson, I. M. S., and Hughes, J. A. (1974). Benefit from adrenocorticotrophin in myasthenia gravis. Journal of Neurology, Neurosurgery, and Psychiatry, 37, 412-415.

Dählback, O., Elmqvist, D., Johns, T. R., Radner, S., and Thesleff, S. (1961). An electrophysiologic study of the neuromuscular junction in myasthenia gravis. Journal of Physiology, 156, 336-343. 